\title{
Papilomavirus bovino tipo 2 en un sarcoide equino en Costa Rica
}

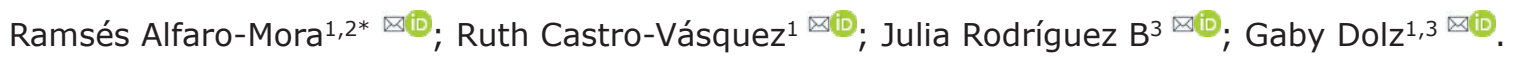

\begin{abstract}
${ }^{1}$ Universidad Nacional, Posgrado Regional en Ciencias Veterinarias Tropicales, Maestría en Enfermedades Tropicales, Heredia, Costa Rica.

2Universidad Latina de Costa Rica, Dirección de Investigación, San José, Costa Rica.

3Universidad Nacional, Escuela de Medicina Veterinaria, Heredia, Costa Rica.

*Correspondencia: ramses.alfaro@ulatina.net
\end{abstract}

Recibido: Julio 2020; Aceptado: Febrero 2021; Publicado: Mayo 2021.

\section{RESUMEN}

Objetivo: Identificar mediante técnicas moleculares la presencia de papilomavirus bovino (BPV) en un sarcoide equino, y realizar la caracterización histológica de este. Materiales y métodos: A partir de lesiones de sarcoide en una yegua de raza pinto americano se realizó la extracción de ácidos nucleicos para su identificación mediante técnicas moleculares. Se amplificó el gen correspondiente al segmento L1 para papilomavirus a través de reacción en cadena de la polimerasa, se secuenció y luego fue analizado utilizando el algoritmo BLAST. Se realizó tinción de hematoxilina-eosina del tejido y se analizó en microscopio de luz 100X. Resultados: Se detectó BPV-2 en la lesión de sarcoide equino; el sarcoide se clasificó como nodular de acuerdo con sus características morfológicas e histológicas. La histopatología mostró una lesión fibrótica, con neoplasia exofítica y expansión del estrato espinoso. Conclusiones: El estudio demostró por primera vez la presencia de BPV-2 en un sarcoide equino en Costa Rica utilizando técnicas moleculares y su asociación con un sarcoide de tipo nodular.

Palabras clave: Equino; Reacción en cadena de la polimerasa; Papilomavirus bovino; fibrosis; Costa Rica (Fuente: DeSC).

\section{ABSTRACT}

Objective. To identify by molecular techniques the presence of bovine papillomavirus (BPV) in an equine sarcoid, and to carry out its histological characterization. Materials and methods. Using a sarcoid lesion from a Pinto Americano mare, nucleic acid extraction and identification by molecular techniques was performed. A partial region of the gene corresponding to the L1 segment of papillomavirus was amplified by polymerase chain reaction, then it was sequenced and analyzed using BLAST algorithm. Hematoxylin-eosin staining of the tissue was performed and analyzed under a 100X light microscope. Results. BPV-2 was detected in the equine sarcoid lesion, and the lesion was classified as nodular according to its morphological and histological characteristics. Histopathology showed a fibrotic lesion, with exophytic neoplasia and expansion of the spinous layer. Conclusions. The study established for first time the presence of BPV-2 in an equine sarcoid in Costa Rica using molecular techniques and its association with a nodular-type sarcoid.

Keywords: Equine; Polymerase chain reaction; Bovine papillomavirus; fibrosis; Costa Rica (Source: MeSH).

Como citar (Vancouver).

Alfaro-Mora R, Castro-Vásquez R, Rodríguez BJ, Dolz G. Papilomavirus bovino tipo 2 en un sarcoide equino en Costa Rica. Rev MVZ Córdoba. 2021; 26(3):e2040. https://doi.org/10.21897/rmvz.2040 


\section{INTRODUCCIÓN}

El sarcoide equino es el tumor más común en la piel de equinos alrededor del mundo. Este tumor no hace metástasis, pero puede ser invasivo, generar defectos cosméticos y llevar a la aparición de úlceras. Si afecta los párpados, puede afectar la visión (1). El desarrollo de estas lesiones en los caballos se asocia a una infección con el Papilomavirus bovino (BPV) aunque una infección del virus por sí sola no es suficiente para desarrollar el tumor $(2,3,4)$. Los sarcoides se clasifican según sus características clínicas, como: ocultos, nodulares, verrugosos, fibroblásticos, mixtos o malignos $(5,6)$.

La mayoría de trabajos que determinaron la presencia de BPV en el sarcoide equino mediante técnicas moleculares se han realizado en Europa. Tanto en Europa como en Australia se ha reportado la presencia del papilomavirus bovino genotipo 1 (BPV-1). En el continente americano en Estados Unidos y Canadá se ha identificado el BPV-2 y en Brasil el BPV-1, BPV-2 y BPV-13 $(6,7,8)$. También en Brasil se reportó la coexistencia de los tres genotipos en un mismo animal de raza equina (8).

Un estudio realizado por Vindas et al (9) en Costa Rica, reporta el tratamiento de cuatro casos de equinos con diagnóstico clínico presuntivo o histopatológico de sarcoide. En ese estudio se determinó, que los dos equinos que recibieron tratamiento combinado de remoción quirúrgica, cauterización y autovacuna, evolucionaron más favorablemente, sin desarrollar recidiva, comparados con los otros dos casos que fueron tratados sin la administración de la vacuna autóloga, recomendando más investigaciones (9). El objetivo del presente trabajo fue identificar mediante técnicas moleculares la presencia de papilomavirus bovino (BPV) en sarcoide equino.

\section{MATERIALES Y MÉTODOS}

Recolecta de muestras: Una yegua de raza Pinto Americano, de 3 años de edad, ubicada en Bajo el Remolino, Buenos Aires de Puntarenas, Costa Rica presentaba desde hace un año tres lesiones tipo sarcoide en el miembro posterior izquierdo a nivel medial (Figura 1) y una lesión tipo sarcoide en la oreja, las cuatro lesiones con características idénticas a nivel macroscópico. De dos lesiones del miembro posterior se tomó una muestra, realizando cortes de $1-2 \mathrm{~cm}$ de longitud. Uno de los cortes se conservó en etanol de 70\% para los análisis moleculares (Figura 1A) y el otro en formaldehido $10 \%$ para análisis histopatológico hasta su procesamiento (Figura 1B).

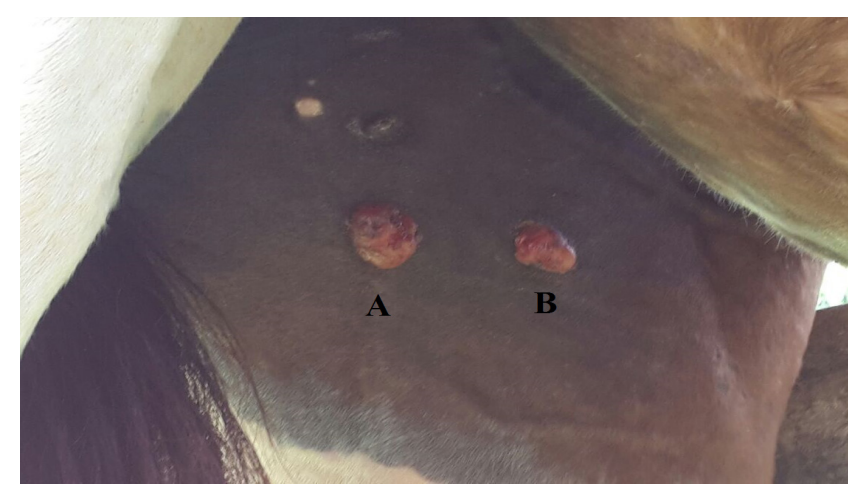

Figura 1. Lesiones tipo sarcoide ubicadas en miembro posterior izquierdo de yegua A. Muestra analizada mediante PCR B. Muestra analizada histopatológicamente.

\section{Análisis molecular (extracción, amplificación} y secuenciación del ADN): La muestra conservada en etanol se sometió a extracción de ADN siguiendo las especificaciones del Kit DNeasy Blood and Tissue (Qiagen, Hilden, Germany). Mediante la técnica de reacción en cadena de la polimerasa (PCR) se amplificó una región conservada de $478 \mathrm{pb}$ del gen que codifica para la proteína estructural L1 (10), usando los cebadores FAP59/FAP64 (5'-TAACWGTIGGICAYCCWTATT3'/5'-CWATATCWVHCATITCICCATC-3'). La reacción de PCR se realizó con DreamTaq Master Mix 1X (Thermo Scientific, USA), 0,25 $\mu \mathrm{M}$ de cada cebador, $1.25 \mu \mathrm{L}$ de ADN (100 ng/uL) y agua libre de nucleasas (Thermo Scientific, USA) en un volumen de $25 \mu \mathrm{L}$. Las condiciones para la amplificación fueron una desnaturalización inicial a $94^{\circ} \mathrm{C}$ de 10 minutos, seguida de 45 ciclos de desnaturalización a $94^{\circ} \mathrm{C}$ por 90 segundos, hibridación a $50^{\circ} \mathrm{C}$ por 90 segundos, extensión 90 segundos a $72^{\circ} \mathrm{C}$; y una extensión final a $72^{\circ} \mathrm{C}$ de 5 minutos (11).

La visualización del producto de PCR se llevó a cabo por electroforesis utilizando un gel de agarosa al $1 \%$ teñido con GelRed (Biotium, USA). El marcador de peso molecular usado fue GeneRuler 1Kb DNA Ladder (Thermo Scientific, USA). El producto de PCR se envió a secuenciar a Macrogen, Seoul, Korea. La edición de la secuencia se realizó con el software Bioedit 7.2.5. Posteriormente se comparó con las secuencias de la base de datos del Genbank por medio del algoritmo BLASTn. También se realizó un alineamiento de la secuencia obtenida 
contra las secuencias reportadas de los 13 genotipos existentes, para establecer el nivel de homología de los amplicones de L1 con el programa ClustalW ${ }^{\circledR}$.

Análisis morfológico e histológico: La muestra conservada en formaldehído se sometió a tinción con hematoxilina-eosina y se analizó en microscopio de luz 100X (12). La lesión se clasificó de acuerdo con Knottenbelt (5), donde se establece seis tipos reconocibles (sarcoide oculto, verrucoso, nodular, fibroblastico, mixto y malevolente) (5).

\section{RESULTADOS}

La secuencia de la proteína estructural L1 amplificada de la muestra del sarcoide equino (número de acceso GenBank MN304951.1) resultó ser $100 \%$ (400/400pb) similar con una secuencia de BPV-2 que había sido aislada de una lesión papilomatosa de una vaca de Japón (número de acceso GenBank MH589273.1) (Figura 2). El alineamiento de la secuencia obtenida con la de los 13 genotipos existentes reveló que hay un $99 \%$ de identidad nucleotídica con el BVP-2 (GenBank M20219.1). Con el resto de genotipos la homología encontrada fue de $49 \%-89 \%$.

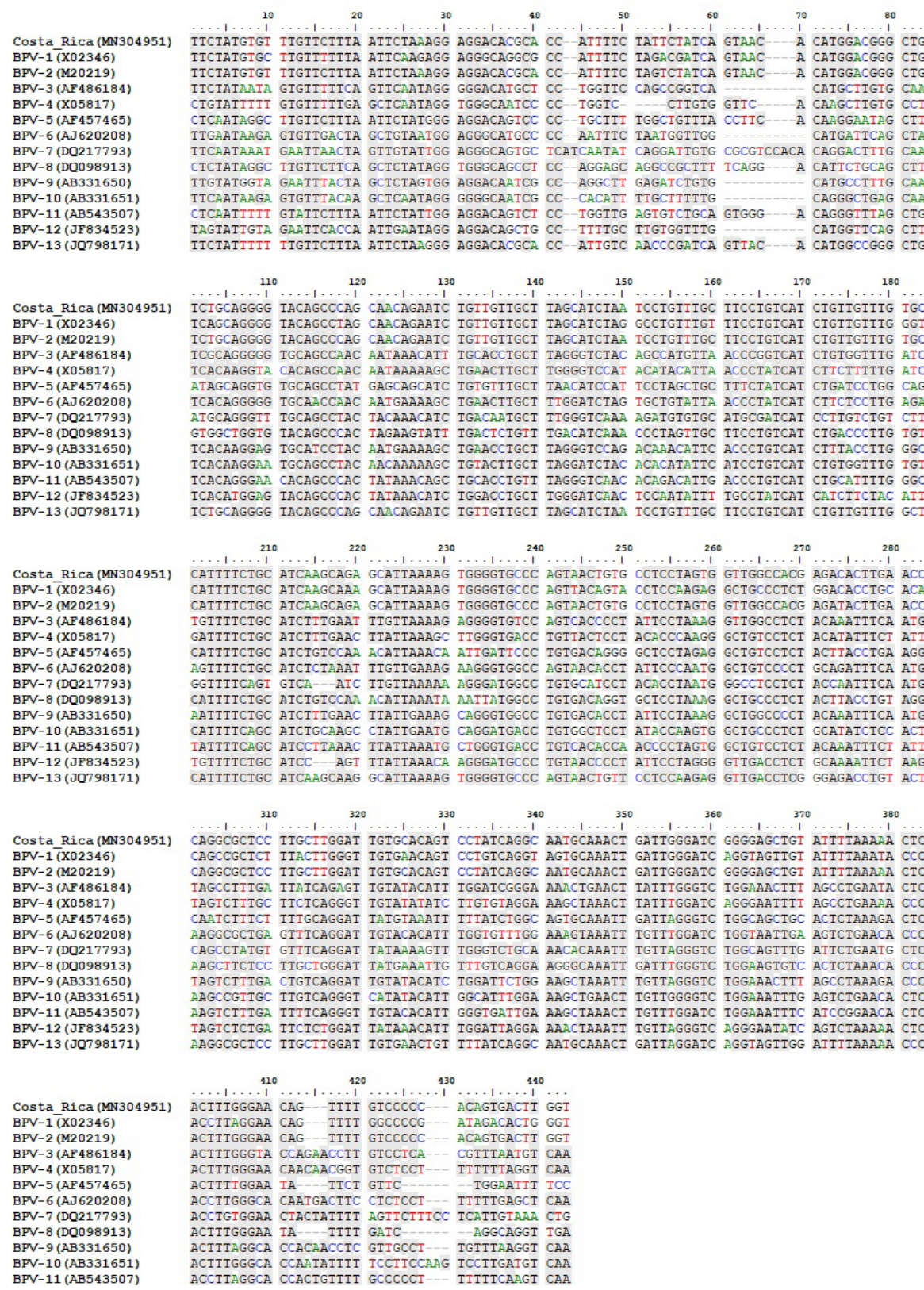

Figura 2. Alineación de la secuencia del gen L1 del sarcoide encontrado en un equino en Costa Rica, con las secuencias de los 13 genotipos existentes de secuencias de papilomavirus bovino. En gris se muestran las regiones idénticas entre secuencias. 
El sarcoide se clasificó como nodular de tipo A de acuerdo a sus características morfológicas e histológicas. El análisis microscópico determinó la lesión como fibroblástica. El estudio histopatológico determinó neoplasia exofítica y expansión del estrato espinoso en algunas áreas de la lesión (Figura 3).

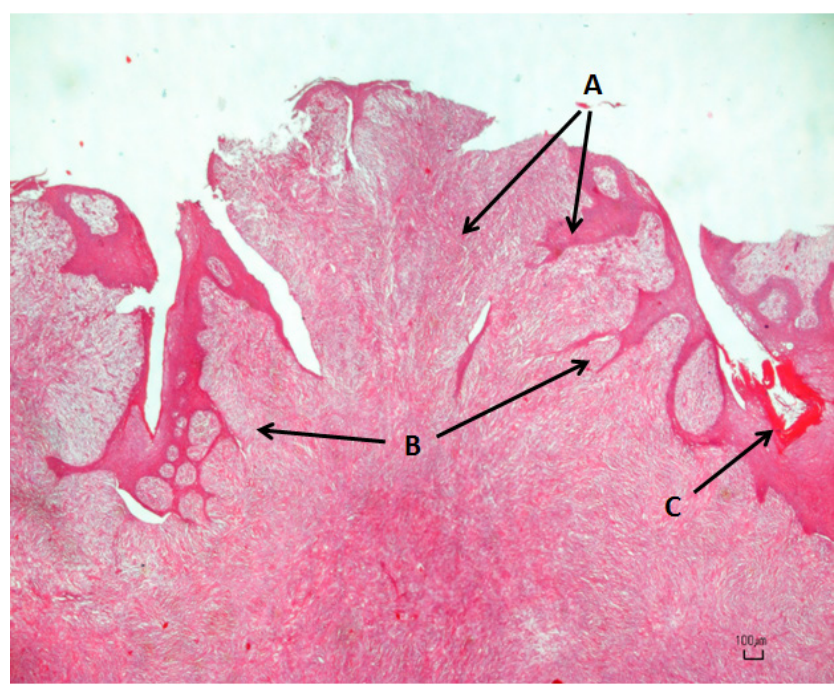

Figura 3. Histopatología del sarcoide equino de la extremidad de la yegua. Mediante la tinción de hematoxilina y eosina se puede observar: (A) Neoplasma exofítico compuesto por células neoplásicas epidérmicas y mesenquinales, (B) Extensiones epiteliales y (C) Expansión de estrato espinoso por hiperqueratosis.

\section{DISCUSIÓN}

El hallazgo de BPV-2 en un sarcoide equino en Costa Rica concuerda con las investigaciones de USA y Brasil, representando además el primer reporte en Costa Rica y Centroamérica (8). La presencia del virus en los sarcoides de los equinos puede explicar los resultados obtenidos por Vindas et al (9), quienes determinaron recidivas en equinos que no recibieron vacuna autóloga en comparación con otros dos animales a los cuales si se les aplicó la autovacuna (9).

La lesión se determinó como fibroblástica en el análisis microscópico, ya que se evidenció alta fibrosis. Las lesiones de tipo fibroblastico que se observaron coinciden con lo que menciona Knottenbelt (5), donde se describen con un aspecto carnoso, y los sitios predilectos en donde suelen manifestarse son: la ingle, párpados, y parte interior de las extremidades (5). Los hallazgos histopatológicos evidenciaron la neoplasia exofítica, mostrando células epidérmicas organizadas en extensiones epiteliales que se proyectan en dirección al tejido conectivo subyacente de la piel (conocidas en inglés como: rete pegs), y expansión del estrato espinoso en algunas áreas de la lesión, considerados hallazgos característicos de los sarcoides $(13,14)$. Según Chambers et al $(4)$, la infección de fibroblastos y la inducción de tumores fibroepiteliales es atribuible al BPV-2 (4). Actualmente no existe ningún estudio que asocie la presencia de un genotipo específico de BPV y la clasificación establecida por Knottenblet (5), así como tampoco relación de los genotipos con sitios donde se manifiestan las lesiones en los equinos.

Aunque la infección por BPV es necesaria para el desarrollo de un sarcoide, se desconoce el mecanismo de desarrollo del sarcoide equino (15). Se cree que una predisposición genética y traumas pueden jugar un papel importante en el desarrollo de las lesiones, ya que se ha demostrado la presencia de BPV en piel sana de equinos $(4,15,16)$. Los estudios que existen sobre el sarcoide equino son pocos, y generalmente se limitan al reporte de casos. Se recomienda realizar estudios más amplios, para determinar el porcentaje de sarcoides equinos con etiología viral y los genotipos presentes en estas lesiones, para recomendar el uso de autovacunas o vacunas homólogas como tratamiento.

En conclusión, se determinó por primera vez la presencia de BPV-2 en un sarcoide equino en Costa Rica. Se recomienda realizar más estudios para determinar la presencia de otros BPV asociados al sarcoide equino y el porcentaje de participación de BPV en los sarcoides equinos de Costa Rica.

\section{Conflicto de Intereses}

No existen conflictos de interés en este estudio, incluidos la preparación del manuscrito y la publicación del artículo.

\section{Agradecimientos}

A la Dra. Elisabetta Antuofermo por su ayuda con las fotografías para el análisis histopatológico y al Sr. Arcelio Mora Piedra por facilitar las muestras analizadas. 


\section{REFERENCIAS}

1. Wobecer BK, Davies JL, Hill JE, Jackson ML, Kidney BA, Mayer MN, Townsend HG, Allen AL. Epidemiology of equine sarcoide in horses in western Canada. Can Vet J. 2010; 51(10):1103-1108. https://pubmed.ncbi. nlm.nih.gov/21197201/

2. Bogaert L, Martens A, De Baere C, Gasthuys F. Detection of bovine paillomavirus DNA on the normal skin and in the habitual surroundings of horses with and withou equine sarcoids. Res Vet Sci. 2005; 79(3):253-258. https:// doi.org/10.1016/j.rvsc.2004.12.003

3. Bogaert L, Van Poucke M, De Baere C, Dewulf J, Peelman L, Ducatelle R, Gasthuys F, Martens A. Bovine papillomavirus load and mRNA expression, cell proliferation and p53 expression in four clinical type of equine sarcoide. J Gen Virol. 2007; 88(Pt 8):2155-2161. https://doi.org/10.1099/ vir.0.82876-0

4. Chambers G, Ellsmore VA, O'Brien M, Reid SW, Love S, Campo MS, Nasir L. Association of bovine papillomavirus with the equine sarcoide. J Gen Virol. 2003; 84(5):10551062. https://doi.org/10.1099/vir.0.18947-0

5. Knottenbelt DC. A Suggested Clinical Classification for the equine sarcoide. Clin Tech Equine Pract. 2005; 4(4):278-295. https:// doi.org/10.1053/j.ctep.2005.10.008

6. Wobeser BK. Making the Diagnosis: Equine Sarcoid. Vet Pathol. 2017; 54(1):9-10. https:// doi.org/10.1177/0300985816664793

7. Lunardi $M$, Kussumoto $B$, Arellano RA, Borges W, Fernandes A, Alcindo A. Bovine Papillomavirus Type 13 DNA in Equine Sarcoids. J Clin Microbiol. 2013; 51(7):21672171. https://doi.org/10.1128/JCM.00371-13

8. de Alcântara BK, Alfieri AA, Headley SA, Rodrigues WB, Otonel R, Lunardi M, Alfieri AF. Molecular characterization of bovine Deltapapillomavirus (BPV1, 2, and 13) DNA in equine sarcoids. Pesq Vet Bras. 2015; 35(4):431-436. https://doi.org/10.1590/ $\underline{\text { S0100-736X2015000500007 }}$

9. Vindas $R$, Villagra $R$, Vargas J, Jiménez $C$, Marín R, Rodríguez H, Estrada M. Tratamiento de sarcoide equino: Reporte de cuatro casos clínicos. Rev Ciencias Veterinarias. 2015; 31(2):59-68. https://www.revistas. una.ac.cr/index.php/veterinaria/article/ view/6863/7035
10. Araldi RP, Carvalho RF, Melo TC, Diniz NS, Sant 'Ana TA, Mazzuchelli-de-Souza J, Spadacci-Morena DD, Beçak W, Stocco RC. Bovine papillomavirus in beef cattle: first description of BPV-12 and putative type BAPV8 in Brazil. Genet Mol Res. 2014; 13(3):5644-5653. https://doi. org/10.4238/2014.July.25.20

11. Roperto $S$, Brun R, Paolini f, Urrro C, Russo V, Borzacchiello G, Pagnini U, Raso C, Rizzo C, Roperto F, Venuti A. Detection of bovine papillomavirus type 2 in the peripheral blood of cattle with urinary bladder tumours: possible biological role. J Gen Virol. 2008; 89(Pt 12):3027-3033. https://doi. org/10.1099/vir.0.2008/004457-0

12. Williams JH, Van E, Nel PJ, Lane, E, Van E, Bengis R, et al. Pathology and inmunohistochemistry of papillomavirusassociated cutaneous lesions in Cape mountain zebras, giraffe, sable antelope and African buffalo in South Africa. JI S. Afr Vet Ass. 2011; 82(2):7-106. https://doi. org/10.4102/jsava.v82i2.42

13. Martens A, De Moor A, Demeulemeester J, Ducatelle R. Histopathological characteristics of fice clinical types of equine sarcoide. Res Vet Sci. 2000; 69:295-300. https://doi. org/10.1053/rvsc.2000.0432

14. Taylor S, Haldorson G. A review of equine sarcoid. Equine Vet. Educ. 2013; 25(4): 210-216. https://doi.org/10.1111/j.20423292.2012.00411.x

15. Bogaert $L$, Marten A, Kast WM, Van $\mathrm{ME}$, De CoCK H. Bovine papillomavirus DNA can be detected in keratinocytes of sarcoid tumors. Vet Microbiol. 2010. 146(34):269-275. https://doi.org/10.1016/j. vetmic.2010.05.032

16. Wobecer BK, Hill J, Jackson M, Kidney B, Mayer $M$, Townsed $H$, et al. Localization of Bovine papillomavirus in equine sarcoids and inflamamatory skin condition of horses using laser microdissection and two forms of DNA amplification. J Vet Diagn Invest. 2012; 24(1):32-41. https://doi. org/10.1177/1040638711425952 